\title{
Aplicação de princípios de bioenergética no cálculo da estequiometria de reações biológicas em processos de tratamento de águas residuárias
}

\section{Application of bioenergetic principles to the stoichiometry of biological reactions in wastewater treatment processes}

\author{
Antônio D. Benetti \\ Doutor em Engenharia Ambiental pela Cornell University, Estados Unidos. Professor Associado do Instituto de \\ Pesquisas Hidráulicas da Universidade Federal do Rio Grande do Sul (UFRGS)
}

\section{Sérgio F. Aquino}

Doutor em Engenharia Química por Imperial College London, Reino Unido. Professor Adjunto do Departamento de Química da Universidade Federal de Ouro Preto (UFOP)

\section{Resumo}

O tratamento biológico de águas residuárias é realizado por microrganismos que utilizam determinado substrato para obtenção de energia e crescimento celular através de reações de oxidação-redução e fermentação. Esses mecanismos podem ser expressos por meio de reações estequiométricas desenvolvidas a partir de princípios da termodinâmica. As reações são dependentes dos compostos doadores e aceptores de elétrons. As equações balanceadas resultantes permitem estimar as necessidades de nutrientes, a variação de pH/alcalinidade, a produção de biomassa e gases e o coeficiente de produção celular associados aos sistemas biológicos de tratamento. Esta nota técnica descreveu a metodologia para cálculo estequiométrico de reações bioquímicas usando princípios de bioenergética, apresentando exemplos para diferentes substratos e aceptores de elétrons.

Palavras-chave: estequiometria de reações biológicas; tratamento biológico de águas residuárias; bioenergética

\begin{abstract}
Biological wastewater treatment is performed by microorganisms by means of oxidation-reduction and fermentation reactions that release energy for growth and maintenance. These processes can be represented by stoichiometric reactions developed based on thermodynamic principles. Reactions are dependent on the compounds used as electron donor and acceptor. The overall stoichiometric reactions allow estimations of nutrient requirements, alkalinity/pH changes, biomass and gas production, and yield coefficient. This article described a methodology for stoichiometry calculations using bioenergetic principles, presenting examples with different substrates and electron acceptors.
\end{abstract}

Keywords: stoichiometry of biological reactions; biological wastewater treatment; bioenergetic.

\section{Introdução}

A estequiometria das reações químicas é ferramenta essencial no desenvolvimento de balanços de massa em reatores utilizados no controle da poluição ambiental. Por meio das equações balanceadas pode-se estimar a quantidade de reagentes e produtos associados a determinado sistema de tratamento. Entretanto, em sistemas biológicos de tratamento a derivação de equações balanceadas é mais complicada quando comparada aos sistemas químicos, devido aos seguintes aspectos: i) as reações microbianas geralmente envolvem oxidação e redução de mais de uma espécie; ii) os micro-organismos são simultaneamente os catalisadores e produtos das reações; iii) os microrganismos realizam as reações bioquímicas para obter energia que será utilizada tanto para a síntese celular quanto para a manutenção das células (RITTMANN \& McCARTY, 2001). Dessa forma, equações globais que representam o tratamento biológico devem levar em conta não só o consumo de reagentes e a geração de produtos químicos, mas também a produção de novas células (biomassa).

O tratamento biológico de águas residuárias baseia-se em reações de oxidação-redução (redox) nas quais uma fração de elétrons do doador é transferida para um aceptor, liberando energia para que uma outra fração de elétrons do doador seja convertida em novas 
células. A Figura 1 ilustra o processo oxidativo microbiano. No caso de fermentação, não há um doador externo de elétrons.

Do ponto de vista matemático, o fluxo representado na Figura 1 é expresso pelas Equações 1 e 2 .

$R=f_{e} \cdot R_{a}+f_{s} \cdot R_{s}-R_{d}$

Equação 1

$\mathrm{f}_{\mathrm{e}}+\mathrm{f}_{\mathrm{s}}=1,0$

Equação 2

sendo:

R: reação global balanceada;

$\mathrm{f}_{\mathrm{e}}$ e $\mathrm{f}_{\mathrm{s} \text { : }}$ frações do doador de elétrons usados para energia e síntese, respectivamente;

$\mathrm{R}_{\mathrm{a} \text { : }}$ semirreação do aceptor de elétrons;

$\mathrm{R}_{\mathrm{s}}$ : semirreação de síntese de células bacterianas;

$\mathrm{R}_{\mathrm{d}}$ : semirreação do doador de elétrons.

A Equação 1 expressa a reação na qual o doador de elétrons é oxidado (representado pelo termo $\mathrm{R}_{\mathrm{d}}$ ), sendo os elétrons liberados usados para reduzir um aceptor de elétrons e produzir biomassa, representados pelos termos $f_{e} \times R_{a}$ e $f_{s} \times R_{s}$, respectivamente. Semirreações são expressas na forma de redução; por essa razão, o termo $R_{d}$ da Equação 1 aparece com sinal invertido.

Dependendo dos compostos doadores e aceptores de elétrons e das frações alocadas para energia (f) e síntese (f $)$, a reação estequiométrica representada pela Equação 1 terá diversas formas. A obtenção de uma reação estequiométrica balanceada é útil para o projeto e operação de estações de tratamento de águas residuárias. Por exemplo, através desta reação, podem ser estimadas a produção de biomassa, os requerimentos de nutrientes, o consumo de alcalinidade, a variação de pH e a produção de biogás.

Esta nota técnica teve como objetivo descrever o procedimento para cálculo da estequiometria de reações aplicada a processos biológicos de tratamento a partir do conceito de bioenergética. A metodologia foi apresentada em trabalhos pioneiros de McCarty (1965, 1971, 1975) com relação à termodinâmica das reações biológicas. Esses conceitos foram progressivamente incorporados na literatura da área de biotecnologia aplicada ao tratamento de resíduos (por exemplo, RITTMANN \& McCARTY, 2001; METCALF \& EDDY, 2003; COMEAU, 2008).

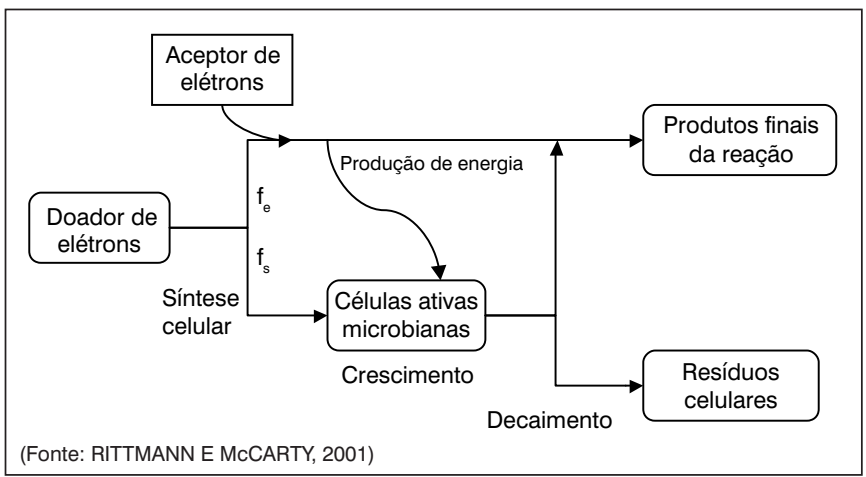

Figura 1 - Produção de energia e síntese celular no processo oxidativo microbiano

\section{Metodologia}

McCarty (1971) introduziu o conceito de equivalente de elétrons (eqe) com o objetivo de simplificar a complexidade das relações químicas envolvidas no processo biológico. Um eqe é definido como a quantidade de uma substância que libera 1,0 mol de elétrons em uma reação de oxidação. No caso particular de doadores de elétrons orgânicos, a definição é dada em termos de oxidação carbonácea total. Assim, 1,0 eqe é a quantidade do composto orgânico que libera 1,0 mol de elétrons ( $e^{-}$) durante a sua oxidação total, com a mineralização do $\mathrm{C}$ e $\mathrm{N}$ orgânicos em $\mathrm{CO}_{2}$ e $\mathrm{NH}_{4}^{+}$.

Para qualquer matéria orgânica, 1,0 eqe equivale a 8,0 g de DQO. Isto é demonstrado pela reação de redução de oxigênio (Equação 3) e pela própria definição da Demanda Química de Oxigênio, que é a quantidade de oxigênio requerida para oxidar a fração carbonácea de um composto orgânico. Pela Equação 3 observa-se que 1/4 mol de $\mathrm{O}_{2}$ (8 g) serão consumidos na aceitação de 1,0 mol e- do doador.

$\frac{1}{4} \mathrm{O}_{2}+\mathrm{H}^{+}+\mathrm{e}^{-} \rightarrow \frac{1}{2} \mathrm{H}_{2} \mathrm{O}$

Equação 3

Para o caso de síntese bacteriana, a definição de eqe depende da fonte de nitrogênio. Por exemplo, a semirreação de síntese tendo amônia como fonte de nitrogênio é dada pela Equação 4.

$$
\begin{array}{r}
\frac{1}{5} \mathrm{CO}_{2}+\frac{1}{20} \mathrm{HCO}_{3}^{-}+\frac{1}{20} \mathrm{NH}_{4}^{+}+\mathrm{H}^{+}+\mathrm{e}^{-} \rightarrow \frac{1}{20} \mathrm{C}_{5} \mathrm{H}_{7} \mathrm{O}_{2} \mathrm{~N}+\frac{9}{20} \mathrm{H}_{2} \mathrm{O} \\
\text { Equação } 4
\end{array}
$$

sendo que $\mathrm{C}_{5} \mathrm{H}_{7} \mathrm{O}_{2} \mathrm{~N}$ representa a composição média de biomassa ativa sintetizada, com massa molar de 113 g (McCarty, 1971). Vale ressaltar que a composição média da biomassa pode ser determinada experimentalmente por meio da análise elementar (em analisadores CHN) de lodos anaeróbios e aeróbios. Dessa forma, de acordo com a Equação 4, 1,0 eqe é igual a 1/20 mol $\mathrm{C}_{5} \mathrm{H}_{7} \mathrm{O}_{2} \mathrm{~N}$, correspondente a 5,65 g de sólidos suspensos voláteis (SSV).

A bioenergética permite quantificar a energia liberada em reações biológicas, que depende dos compostos que servem como doador e aceptor de elétrons. Vários compostos orgânicos e inorgânicos podem servir como doadores de elétrons (substrato), constituindo-se na fonte de alimento dos organismos envolvidos na reação biológica. Os principais compostos que servem como aceptores de elétrons em sistemas biológicos são oxigênio, nitrato, nitrito, ferro (III), sulfato e dióxido de carbono.

A energia liberada em uma reação redox pode ser calculada através da variação da Energia Livre de Gibbs $\left(\Delta G^{\circ}\right)$. O sobrescrito indica que os reagentes e produtos envolvidos na reação encontram-se na condição padrão, ou seja, a $25^{\circ} \mathrm{C}$ e atividade unitária (aproximadamente $1 \mathrm{~mol} / \mathrm{L}$ para solutos, pressão parcial de 1 atm para gases). No caso de sistemas biológicos, McCarty (1975) fez uma correção no valor de $\Delta G^{\circ}$ para $\mathrm{pH} 7,0$, sendo que uma lista extensa de valores 
de $\Delta \mathrm{G}^{\circ}$ para semirreações de síntese bacteriana, de acepção e doação de elétrons é apresenta por Sawyer et al. (2003). A substituição de semirreações apropriadas, na Equação 1, resultará em uma reação global normalizada para 1,0 eqe.

A transferência de energia do substrato (doador de elétrons) para síntese e manutenção biológica é realizada em duas etapas. Na primeira, o substrato transfere energia para um transportador, representado por adenosina difosfato (ADP), com formação de um transportador "carregado" de energia, adenosina trifosfato (ATP). A energia armazenada no ATP é usada, então, em reações para síntese e manutenção celular. Em cada etapa do processo de transferência há uma perda de energia na forma de calor, de forma que a fração de energia efetivamente usada varia entre 0,4 e 0,8, sendo 0,6 o valor típico estimado (McCARTY, 1971)

Considerando que uma parte da energia liberada da reação redox é perdida na transferência para o transportador de energia (ATP), pode-se determinar que a energia disponível para a reação de síntese é dada pela Equação 5 (COMEAU, 2008).

$\Delta \mathrm{G}_{\text {cat }}=\mathrm{k} \cdot \Delta \mathrm{G}_{\mathrm{r}}$

sendo:

$\Delta \mathrm{G}_{\text {cat }}$ : energia livre de Gibbs disponibilizada pelo catabolismo de 1,0 eqe do doador de elétrons;

$\mathrm{k}$ : fração da energia efetivamente capturada pelo transportador (tipicamente, 0,60);

$\Delta G_{\mathrm{r}}$ : variação de energia livre da reação.

McCarty (1971) postulou que a energia requerida para converter as fontes de carbono e nitrogênio em células $\left(\Delta G_{s}\right)$ consiste de três frações de energia (Equação 6)

$$
\Delta G_{s}=\frac{\Delta G_{p}}{k^{m}}+\Delta G_{c}+\frac{\Delta G_{n}}{k}
$$

sendo:

$\Delta \mathrm{G}_{\mathrm{s}}$ : energia livre requerida para síntese (anabolismo) de 1,0 eqe de células (kJ/eqe).

$1^{\circ}$ ) Energia requerida para converter a fonte de carbono em um composto intermediário, piruvato, considerando que este ocupa uma posição central dos vários caminhos metabólicos. Assim, $\Delta G_{p}=$ energia livre requerida (ou liberada) na conversão da fonte de carbono em piruvato. Se energia for liberada, $\Delta G_{\mathrm{p}}$ será menor que zero e $\mathrm{m}=$ -1; se houver consumo de energia para oxidação a piruvato, $\Delta G_{p}$ será maior que zero e $\mathrm{m}=+1$

$2^{\circ}$ ) Energia requerida para converter 1,0 eqe de piruvato em células bacterianas, representada por $\Delta G_{c}$. Esse valor é constante, 31,41 kJ/eqe células.

$3^{\circ}$ ) Energia requerida para reduzir uma fonte oxidada de nitrogênio em amônia, antes da síntese celular, representada por $\Delta G_{n}$. Os valores desta energia, calculados por McCarty (1971), são, respectivamente,
17,46; 13,61 e 15,85 kJ/eqe células para $\mathrm{NO}_{3}^{-}, \mathrm{NO}_{2}^{-}$e $\mathrm{N}_{2}$. Quando amônia é a fonte de nitrogênio, $\Delta G_{n}$ é igual a zero.

Considerando-se que a energia consumida no anabolismo (síntese) é igual a energia aproveitada do catabolismo, pode-se escrever a Equação 7.

$-\mathrm{f}_{\mathrm{s}} \cdot \Delta \mathrm{G}_{\mathrm{ana}}=\mathrm{f}_{\mathrm{e}} \cdot \Delta \mathrm{G}_{\text {cat }}$

Equação 7

Mas como $\Delta \mathrm{G}_{\text {ana }}=\Delta \mathrm{G}_{\mathrm{s}}$ e $\Delta \mathrm{G}_{\text {cat }}=\mathrm{k} \times \Delta \mathrm{G}_{\mathrm{r}}$, a Equação 7 fica:

$-f_{s} \cdot \Delta G_{s}=f_{e} \cdot k \cdot \Delta G_{r}$

Equação 8

O sinal negativo no lado esquerdo da Equação 8 representa o fato de que a síntese consome energia. A Equação 8 pode ser reescrita na forma da Equação 9, e em conjunto com as Equações 10 e 2 permitem o cálculo de $\mathrm{f}_{\mathrm{s}}$ e de $\mathrm{f}_{\mathrm{e}}$, que indicam, respectivamente, a fração de elétrons do doador canalizada para a síntese e geração de energia.

$\frac{f_{e}}{f_{s}}=-\frac{\Delta G_{s}}{k \cdot \Delta G_{r}}$

Equação 9

$$
f_{s}=\frac{1}{1+\left(\frac{f_{e}}{f_{s}}\right)}
$$

Equação 10

\section{Resultados}

A seguir é apresentado o cálculo de reação estequiométrica com uso do conceito de bioenergética. Trata-se, no exemplo, de metabolismo aeróbio no qual esgoto doméstico serve como doador de elétrons.

- Doador de elétrons: esgoto doméstico, representado por $\mathrm{C}_{10} \mathrm{H}_{10} \mathrm{O}_{3} \mathrm{~N}$.

- Aceptor de elétrons: oxigênio $-\mathrm{O}_{2}$

- Fonte de nitrogênio: amônia $-\mathrm{NH}_{4}^{+}$

a) Cálculo de $\Delta G_{r}$ (variação da energia livre de Gibbs da reação) Semirreação de oxidação do doador de elétrons:

$\frac{1}{50} \mathrm{C}_{10} \mathrm{H}_{19} \mathrm{O}_{3} \mathrm{~N}+\frac{9}{25} \mathrm{H}_{2} \mathrm{O} \rightarrow \frac{9}{50} \mathrm{CO}_{2}+\frac{1}{50} \mathrm{HCO}_{3}^{-}+\frac{1}{50} \mathrm{NH}_{4}^{+}+\mathrm{H}^{+}+\mathrm{e}^{-}$ $\Delta \mathrm{G}^{\circ}=-31,80 \mathrm{~kJ} / \mathrm{eqe}$

Semirreação de redução do aceptor de elétrons:

$\frac{1}{4} \mathrm{O}_{2}+\mathrm{H}^{+}+\mathrm{e}^{-} \rightarrow \frac{1}{2} \mathrm{H}_{2} \mathrm{O}$ $\Delta \mathrm{G}^{\circ}=-78,72 \mathrm{~kJ} / \mathrm{eqe}$

Reação global

$\frac{1}{50} \mathrm{C}_{10} \mathrm{H}_{19} \mathrm{O}_{3} \mathrm{~N}+\frac{1}{4} \mathrm{O}_{2} \rightarrow \frac{9}{50} \mathrm{CO}_{2}+\frac{1}{50} \mathrm{HCO}_{3}^{-}+\frac{1}{50} \mathrm{NH}_{4}^{+}+\frac{16}{25} \mathrm{H}_{2} \mathrm{O}$

$\Delta \mathrm{G}^{\mathrm{o}}=-110,52 \mathrm{~kJ} /$ eqe 
sendo:

$\Delta G^{\circ}=\Delta G_{r}=$ energia livre de Gibbs liberada pela reação. Observase que a reação é favorável do ponto de vista termodinâmico $\left(\Delta G^{\circ}<0\right)$.

b) Cálculo de $\Delta \mathrm{G}_{\mathrm{s}}$ (energia requerida para síntese)

O valor de $\Delta G_{s}$ é calculado pela Equação 6 , sendo o valor de $\Delta G_{c}$ constante e igual a 31,41 kJ/eqe células. Como a amônia é a fonte de nitrogênio, o valor de $\Delta \mathrm{G}_{\mathrm{n}}$ é igual a zero. $\mathrm{O}$ valor de $\Delta \mathrm{G}_{\mathrm{p}}$ é calculado somando-se a semirreação de oxidação do substrato (esgoto doméstico) e a semirreação de redução do piruvato.

Semirreação de oxidação do doador de elétrons:

$\frac{1}{50} \mathrm{C}_{10} \mathrm{H}_{19} \mathrm{O}_{3} \mathrm{~N}+\frac{9}{25} \mathrm{H}_{2} \mathrm{O} \rightarrow \frac{9}{50} \mathrm{CO}_{2}+\frac{1}{50} \mathrm{HCO}_{3}^{-}+\frac{1}{50} \mathrm{NH}_{4}^{+}+\mathrm{H}^{+}+\mathrm{e}^{-}$

$\Delta \mathrm{G}^{\mathrm{o}}=-31,80 \mathrm{~kJ} / \mathrm{eqe}$

Semirreação de redução do piruvato:

$\frac{1}{5} \mathrm{CO}_{2}+\frac{1}{10} \mathrm{HCO}_{3}^{-}+\mathrm{H}^{+}+\mathrm{e}^{-} \rightarrow \frac{1}{10} \mathrm{CH}_{3} \mathrm{COCOO}^{-}+\frac{2}{5} \mathrm{H}_{2} \mathrm{O}$

$\Delta \mathrm{G}^{\mathrm{o}}=+35,10 \mathrm{~kJ} / \mathrm{eqe}$

Reação global

$\frac{1}{50} \mathrm{C}_{10} \mathrm{H}_{19} \mathrm{O}_{3} \mathrm{~N}+\frac{1}{50} \mathrm{CO}_{2}+\frac{2}{25} \mathrm{HCO}_{3}^{-} \rightarrow \frac{1}{10} \mathrm{CH}_{3} \mathrm{COCOO}^{-}+\frac{1}{50} \mathrm{NH}_{4}^{+}+\frac{1}{25} \mathrm{H}_{2} \mathrm{O}$

$\Delta \mathrm{G}^{\mathrm{o}}=+3,3 \mathrm{~kJ} / \mathrm{eqe}$

Assim, a conversão de esgoto doméstico em piruvato consome 3,3 kJ/eqe $\left(=\Delta G_{p}\right)$.

A energia requerida para síntese de 1,0 eqe de células bacterianas é calculada substituindo-se os respectivos valores na Equação 6.

$\Delta \mathrm{G}_{\mathrm{s}}=\frac{3,3}{0,6^{1}}+31,41+\frac{0}{0,6}=36,91 \mathrm{~kJ} /$ eqe

Para calcular as frações do doador de elétrons usadas para energia e síntese, usam-se as Equações 2, 9 e 10.

$\frac{f_{e}}{f_{s}}=-\frac{\Delta G_{s}}{k \cdot \Delta G_{r}}=-\frac{36,91}{0,6 \cdot(-110,52)}=0,557$

$\mathrm{f}_{\mathrm{s}}=\frac{1}{1+\left(\frac{\mathrm{f}_{\mathrm{e}}}{\mathrm{f}_{\mathrm{s}}}\right)}=\frac{1}{1+0,557}=0,642$

$\mathrm{f}_{\mathrm{e}}+\mathrm{f}_{\mathrm{s}}=1,0 \therefore \mathrm{f}_{\mathrm{e}}=0,358$

Para o metabolismo desse exemplo, 64,2 e 35,8\% do doador de elétrons serão utilizados, respectivamente, para síntese de novas células e produção de energia. Nesse caso, toda energia liberada foi alocada para síntese. É possível estender o procedimento para considerar, também, a energia requerida para manutenção da célula.
Substituindo-se os valores de $\mathrm{f}_{\mathrm{e}}$ e $\mathrm{f}_{\mathrm{s}}$ na Equação 1, tem-se:

$\mathrm{R}=0,358 \cdot \mathrm{R}_{\mathrm{a}}+0,642 \cdot \mathrm{R}_{\mathrm{s}}-\mathrm{R}_{\mathrm{d}}$

Equação 11

A equação 11 é então usada para se obter as equações específicas de doação e aceitação de elétrons, e de síntese de biomassa.

Equação de aceitação de elétrons (oxigênio como aceptor de elétrons):

$0,358 \cdot \mathrm{R}_{\mathrm{a}}=0,358 \cdot\left(\frac{1}{4} \mathrm{O}_{2}+\mathrm{H}^{+}+\mathrm{e}^{-}=\frac{1}{2} \mathrm{H}_{2} \mathrm{O}\right)$

$0,0895 \mathrm{O}_{2}+0,358 \mathrm{H}^{+}+0,358 \mathrm{e}^{-} \rightarrow 0,1790 \mathrm{H}_{2} \mathrm{O}$

Equação de síntese de biomassa (amônia como fonte de nitrogênio para síntese):

$0,642 \cdot \mathrm{R}_{\mathrm{s}}=0,642 \cdot\left(\frac{1}{5} \mathrm{CO}_{2}+\frac{1}{20} \mathrm{HCO}_{3}^{-}+\frac{1}{20} \mathrm{NH}_{4}^{+}+\mathrm{H}^{+}+\mathrm{e}^{-} \rightarrow \frac{1}{20} \mathrm{C}_{5} \mathrm{H}_{7} \mathrm{O}_{2} \mathrm{~N}+\frac{9}{20} \mathrm{H}_{2} \mathrm{O}\right)$

$0,1284 \mathrm{CO}_{2}+0,0321 \mathrm{HCO}_{3}^{-}+0,0321 \mathrm{NH}_{4}^{+}+0,642 \mathrm{H}^{+}+0,642 \mathrm{e}^{-} \rightarrow 0,0321 \mathrm{C}_{5} \mathrm{H}_{7} \mathrm{O}_{2} \mathrm{~N}+$ $+0,2889 \mathrm{H}_{2} \mathrm{O}$

Equação de doação de elétrons (esgoto doméstico como doador de elétrons):

$-\mathrm{R}_{\mathrm{d}}=0,02 \mathrm{C}_{10} \mathrm{H}_{19} \mathrm{O}_{3} \mathrm{~N}+0,36 \mathrm{H}_{2} \mathrm{O} \rightarrow 0,18 \mathrm{CO}_{2}+0,02 \mathrm{HCO}_{3}^{-}+0,02 \mathrm{NH}_{4}^{+}+\mathrm{H}^{+}+\mathrm{e}^{-}$

Substituindo-se as equações específicas na Equação 11, obtém-se a equação global balanceada para a síntese de 1,0 eqe de biomassa a partir da oxidação de esgoto doméstico por oxigênio molecular.

$0,02 \mathrm{C}_{10} \mathrm{H}_{19} \mathrm{O}_{3} \mathrm{~N}+0,0895 \mathrm{O}_{2}+0,0121 \mathrm{HCO}_{3}^{-}+0,0121 \mathrm{NH}_{4}^{+}$
$0,0321 \mathrm{C}_{5} \mathrm{H}_{7} \mathrm{O}_{2} \mathrm{~N}+0,0516 \mathrm{CO}_{2}+0,1079 \mathrm{H}_{2} \mathrm{O}$$\rightarrow$ Equação 12

A Equação 12 pode ser normalizada para o consumo de 1,0 mol de esgoto doméstico multiplicando-se os coeficientes estequiométricos por 50 (Equação 13).

$1,0 \mathrm{C}_{10} \mathrm{H}_{19} \mathrm{O}_{3} \mathrm{~N}+4,475 \mathrm{O}_{2}+0,605 \mathrm{HCO}_{3}^{-}+0,605 \mathrm{NH}_{4}^{+} \rightarrow 1,605 \mathrm{C}_{5} \mathrm{H}_{7} \mathrm{O}_{2} \mathrm{~N}+$ $+2,580 \mathrm{CO}_{2}+5,395 \mathrm{H}_{2} \mathrm{O}$

Equação 13

O procedimento acima descrito pode ser repetido para outros substratos e aceptores de elétrons. Por exemplo, as Equações 14 a 16 representam as reações estequiométricas nas quais os doadores e aceptores de elétrons são, respectivamente, acetato e nitrato; proteína e sulfato; e carboidrato e dióxido de carbono.

$1,0 \mathrm{CH}_{3} \mathrm{COO}^{-}+1,010 \mathrm{NO}_{3}^{-}+1,010 \mathrm{H}^{+} \rightarrow 0,441 \mathrm{~N}_{2}+0,358 \mathrm{CO}_{2}+$ $+1,0 \mathrm{HCO}_{3}^{-}+0,128 \mathrm{C}_{5} \mathrm{H}_{7} \mathrm{O}_{2} \mathrm{~N}+1,056 \mathrm{H}_{2} \mathrm{O}$

Equação 14 
$1,0 \mathrm{C}_{16} \mathrm{H}_{24} \mathrm{O}_{5} \mathrm{~N}_{4}+7,00 \mathrm{SO}_{4}^{2-}+0,493 \mathrm{HCO}_{3}^{-}+14,487 \mathrm{H}^{+} \rightarrow 0,493 \mathrm{C}_{5} \mathrm{H}_{7} \mathrm{O}_{2} \mathrm{~N}+$ $+3,50 \mathrm{H}_{2} \mathrm{~S}+3,50 \mathrm{HS}^{-}+5,493 \mathrm{H}_{2} \mathrm{O}+13,987 \mathrm{CO}_{2}+3,493 \mathrm{NH}_{4}^{+}$

Equação 15

$1,0 \mathrm{CH}_{2} \mathrm{O}+0,055 \mathrm{HCO}_{3}^{-}+0,055 \mathrm{NH}_{4}^{+}=0,055 \mathrm{C}_{5} \mathrm{H}_{7} \mathrm{O}_{2} \mathrm{~N}+0,362 \mathrm{CH}_{4}+$ $+0,417 \mathrm{CO}_{2}+0,222 \mathrm{H}_{2} \mathrm{O}$

Equação 16

\section{Discussão}

As Equações 13 a 16 representam reações balanceadas de consumo de 1,0 mol de quatro diferentes substratos (doadores de elétrons) e aceptores de elétrons. Essas reações contêm informações muito úteis. Por exemplo, de acordo com a Equação 13, a oxidação de 1,0 mol de esgoto doméstico por oxigênio consume alcalinidade (0,605 mols de $\mathrm{HCO}_{3}^{-}$ou seja, $201 \mathrm{~g}$ de esgoto doméstico consumem 36,9 g de bicarbonato), com provável redução de $\mathrm{pH}$. Também são necessários 0,605 mols de $\mathrm{NH}_{4}^{+}$por cada mol de esgoto oxidado. Já no exemplo da desnitrificação (metabolismo anóxico), o pH deve aumentar devido a utilização de íons hidrogênio e produção de alcalinidade. Em resumo, os cálculos de bioenergética apresentados permitem obter equações globais do metabolismo que fornecem as relações estequiométricas necessárias para o cálculo racional da quantidade de nutrientes, aceptor de elétrons e alcalinidade para o adequado funcionamento dos reatores biológicos.

A estequiometria permite, também, a estimativa do coeficiente de produção celular verdadeiro. Para o exemplo do metabolismo aeróbio (Equação 13), a oxidação total (DQO) de 1,0 mol de esgoto doméstico ou $\mathrm{C}_{10} \mathrm{H}_{19} \mathrm{O}_{3} \mathrm{~N}$ (201 g) requer 12,5 mols de oxigênio (400 g) (Equação 17).

$\mathrm{C}_{10} \mathrm{H}_{19} \mathrm{O}_{3} \mathrm{~N}+12,5 \mathrm{O}_{2} \rightarrow 10 \mathrm{CO}_{2}+8 \mathrm{H}_{2} \mathrm{O}+\mathrm{NH}_{3}$ Equação 17

Assim, 1,0 mol de $\mathrm{C}_{10} \mathrm{H}_{19} \mathrm{O}_{3} \mathrm{~N}$ é equivalente a $400 \mathrm{~g}$ DQO. Da Equação 13, observa-se que 1,0 mol de $\mathrm{C}_{10} \mathrm{H}_{19} \mathrm{O}_{3} \mathrm{~N}$ produz 1,605 mols de $\mathrm{C}_{5} \mathrm{H}_{7} \mathrm{O}_{2} \mathrm{~N}(181,4 \mathrm{~g})$. Assim, o coeficiente de produção celular será:

$\mathrm{Y}=\frac{181,4 \mathrm{gSSV}}{400 \mathrm{~g} \text { DQO }}=0,453 \frac{\mathrm{gSSV}}{\mathrm{gDQO}}$

O coeficiente de produção celular também pode ser estimado com a Equação 18 (COMEAU, 2008).

$\mathrm{Y}=\frac{\mathrm{f}_{\mathrm{s}} \cdot \mathrm{M}_{\mathrm{c}}}{8 \cdot \mathrm{n}_{\mathrm{e}}}$

Equação 18

sendo:

$\mathrm{f}_{\mathrm{s}}$ : fração do doador de elétrons convertido em células [g DQO celular/g DQO consumida];

$\mathrm{M}_{\mathrm{c}}$ : massa de células/mol células $[\mathrm{g} / \mathrm{mol}]$;

8: massa de DQO por eqe do substrato [g DQO/eqe]; $\mathrm{n}_{\mathrm{e}}$ : número de eqe por mol de células. No caso do metabolismo aeróbio com amônia como fonte de Nitrogênio, $\mathrm{n}_{\mathrm{e}}$ é igual a 20 eqe/ mol $\mathrm{C}_{5} \mathrm{H}_{7} \mathrm{O}_{2} \mathrm{~N}$. A massa de um mol de $\mathrm{C}_{5} \mathrm{H}_{7} \mathrm{O}_{2} \mathrm{~N}$ é $113 \mathrm{~g}\left(\mathrm{M}_{\mathrm{c}}\right)$, e no exemplo dado, f é 0,642 g DQO celular/g DQO. Substituindo-se os respectivos valores na Equação 18, obtém-se:

$\mathrm{Y}=\frac{(0,642 \mathrm{~g} \text { DQO celular } / \mathrm{g} \mathrm{DQO} \text { usada }) \cdot\left(113 \mathrm{gSSV} / \mathrm{molC}_{5} \mathrm{H}_{7} \mathrm{O}_{2} \mathrm{~N}\right)}{(8 \mathrm{gDQO} / \text { eqe }) \cdot\left(20 \mathrm{eqe} / \mathrm{molC}_{5} \mathrm{H}_{7} \mathrm{O}_{2} \mathrm{~N}\right)}=0,453 \frac{\mathrm{gSSV}}{\mathrm{gDQO}}$

O valor de Y pode ser usado para estimar a produção de biomassa nos reatores biológicos, servindo assim como um parâmetro para projeto de unidades de tratamento do lodo de excesso gerado em estações de tratamento de efluentes. Como o valor de Y é diretamente proporcional ao valor de $\mathrm{f}_{\mathrm{s}}$, os sistemas que canalizam maior quantidade de elétrons para a redução do aceptor final de elétrons, ou seja, que tem valor de $\mathrm{f}_{\mathrm{e}}$ elevado, produzem pouco lodo e têm baixo valor de Y. Esse é o caso dos sistemas anaeróbios metanogênicos que, por canalizarem grande parte dos elétrons para a redução do dióxido de carbono $\left(\mathrm{CO}_{2}\right)$, produzem um composto altamente energético (metano, $\mathrm{CH}_{4}$ ) que pode ser utilizado como combustível de origem não-fóssil. Como pode ser depreendido da Equação 16, os cálculos de bioenergética apresentados permitem ainda determinar a produção e composição do biogás gerado nos sistemas anaeróbios

Outra informação importante é a variação de energia que é produzida nos diferentes metabolismos. Na reação de oxidação do esgoto doméstico pelo oxigênio, a energia livre liberada é de -110,52 kJ/eqe. Caso os aceptores fossem $\mathrm{NO}_{3}^{-}, \mathrm{SO}_{4}^{2-}$ e $\mathrm{CO}_{2}$, as variações de energia livre seriam -104,00, - 10,95 e -8,28 kJ/eqe (cálculos não mostrados). Como era de se esperar, o metabolismo aeróbio libera mais energia que o anóxico e o anaeróbio. Os micro-organismos heterotróficos que conseguirem obter a maior energia possível nas reações metabólicas terão maior taxa de crescimento e serão dominantes; dessa forma, o metabolismo aeróbio prevalecerá sobre os outros em ambientes com disponibilidade de oxigênio dissolvido (RITTMANN \& McCARTY, 2001).

\section{Conclusões}

Neste artigo foi apresentado um método para cálculo da estequiometria de reações biológicas baseado em princípios de bioenergética, conforme introduzido por McCarty (1965, 1971, 1975). O método permite determinar o consumo/produção de alcalinidade, as necessidades de nutrientes, a produção de biomassa e de biogás, além do coeficiente de produção celular. Os exemplos de aplicação mostram coerência entre as informações fornecidas pelas equações globais balanceadas e o conhecimento prático dos processos biológicos que empregam diferentes metabolismos. Essas informações são úteis para o projeto e operação de estações de tratamento de águas residuárias que utilizam processos biológicos. A validade das equações 
desenvolvidas pode ser verificada experimentalmente em reações com diferentes substratos e aceptores de elétrons.

A metodologia apresentada pode ser estendida para os casos em que haja múltiplos doadores e aceptores de elétrons. Para efluentes complexos, o uso de técnicas como a análise elementar pode ser útil, uma vez que permite determinar a composição, em termos de C, N, H, P, S, de amostras de esgoto, lodo e resíduo a ser tratado.

\section{Referências}

COMEAU, Y. Microbial metabolism. In: HENZE, M.; van LOODSDRECHT, C. M.; EKAMA, G. A.; BRDJANOVIC, D. (eds.) Biological wastewater treatment: principles, modeling and design. London: IWA/Cambridge University Press, cap. 2, p. 9-32, 2008.

McCARTY, P. L. Thermodynamics of biological synthesis and growth. International Journal of Air and Water Pollution, v. 9, p. 621-639, 1965.

Energetics and bacterial growth. In: FAUST, S. D.; HUNTER, J. V. (eds.) Organic compounds in aquatic environments. New York: Marcel Dekker, cap. 21, p. 495-531, 1971.
Stoichiometry of biological reactions. Progress in Water Technology, v. 7, n. 1, p. 157-172, 1975.

METCALF \& EDDY, Inc. Wastewater engineering: treatment and reuse. 4th ed. New York: McGraw-Hill, 2003.

RITTMANN, B. E.; McCARTY, P. L. Environmental biotechnology: principles and applications. Boston: McGraw-Hill, 2001.

SAWYER, C. N.; McCARTY, P. L.; PARKIN, G. F. Chemistry for environmental engineering and science. $5^{\text {th }}$ ed. Boston: McGraw-Hill, 2003. 